\title{
Políticas públicas, estructuras del Estado y defensa del derecho a la protección de la salud
}

\author{
Enrique Ruelas-Barajas, MC, MAP, MHSc, ${ }^{(1)}$ Juan Gabriel Gay-Molina, MC, MPH. ${ }^{(2)}$
}

\author{
Ruelas-Barajas E, Gay-Molina JG. \\ Políticas públicas, estructuras del Estado \\ y defensa del derecho a la protección de la salud. \\ Salud Publica Mex 2008;50 supl 3:S343-S347.
}

\section{Resumen}

Si bien existen estructuras del Estado, que no sólo de gobierno, cuya misión es impulsar y proteger los derechos ciudadanos, es claro que ésta no siempre se cumple cabalmente. Desde la formulación de leyes, políticas públicas, y regulaciones que deben hacer efectivas ambas, y entre todas éstas y su ejecución para asegurar el impacto deseado, existen con frecuencia brechas que terminan por hacerlas inoperantes. El caso de las medidas que a lo largo de muchos años se han establecido para el control del tabaco en México es un claro ejemplo de ello. En efecto, en múltiples ocasiones en el pasado se tomaron medidas de diversa índole que por una u otra razón han sido inefectivas. El postulado central de este ensayo es que desde el diseño de una ley, una política pública, y el logro de su propósito, intervienen necesariamente los fundamentos sobre los cuales se construyó esa política, su pertinencia a las necesidades que le dieron origen y las estructuras que la crean y/o las que deben hacerse responsables no sólo de acatar la ley y de convertirla en una política operativa sino de asegurar que esa operatividad se concrete en logros demostrables en los plazos esperados y con los recursos necesarios. El Consejo de Salubridad General se fortalece y rescata su vocación primigenia para responder como una de las estructuras del Estado que debe contribuir, con otras más, a la articulación de la génesis, formulación y ejecución de políticas públicas para que éstas transiten de un desideratum hacia una realidad de efectos positivos demostrables.

Palabras clave: tabaco; políticas públicas; derechos civiles; México
Ruelas-Barajas E, Gay-Molina JG. Public policy, structures of the state and the advocacy of the right to health protection. Salud Publica Mex 2008;50 suppl 3:S343-S347.

\begin{abstract}
Not always public policies for the advocacy of citizen rights meet their goal, among other things, because between their formulation and their implementation there is a lack of appropriate structures to generate, articulate and execute these policies. This has been the case of Mexico regarding the advocacy of the rights of non smokers. Very few has been achieved in this matter in the past. This article lists some of the potential causes of the lack of implementation of public policies. At the same time, explores the highly dynamic nature of the health care system at the present and towards the future. The magnitude of the challenges faced by the health care system requires concerted actions of many actors, not only from the very health care system but also from outside. Thus, public policies have to be considered not just at the governmental level but at the state level. Therefore, the main argument of this paper is that given the need to respond to complex challenges to protect the health of the population and their rights in a very complex context, there is a need to formulate and implement state policies that require the intervention of the appropriate state structures to make sure that the design and execution leads to the expected achievements. One such structure at the state level, among others, is the General Health Council, an organization established in I84I, that was incorporated within the Mexican Constitution in 1917, depending directly from the President of Mexico as Head of State. This Council has been reinforced to improve its assigned role to better participate, along other state structures, in the formulation and implementation of public policies such as those for the advocacy of citizen rights.
\end{abstract}

Key words: tobacco; public policy; civil rights; Mexico

(I) Secretario del Consejo de Salubridad General. México.

(2) Director de Enfoque en Calidad y Eficiencia en Salud. Consejo de Salubridad General. México.

Fecha de aceptado: 28 de abril de 2008 Solicitud de sobretiros: Dr. Juan Gabriel Gay-Molina. Consejo de Salubridad General, Lieja No. 7, col. Juárez, 06696 México DF. Correo electrónico: jgay@salud.gob.mx 
$\mathrm{S}_{\mathrm{s}}^{\mathrm{i}}$ bien existen estructuras del Estado, que no sólo de gobierno, cuya misión es impulsar y proteger los derechos ciudadanos, es claro que ésta no siempre se cumple cabalmente. Desde la formulación de leyes, políticas públicas y regulaciones que deben hacer efectivas ambas, $y$ entre todas éstas y su ejecución para asegurar el impacto deseado, existen con frecuencia brechas que terminan por hacerlas inoperantes. El caso de las medidas que a lo largo de muchos años se han establecido para el control del tabaco en México es un claro ejemplo de ello. En múltiples ocasiones en el pasado se tomaron medidas de diversa índole que por una u otra razón han sido inefectivas como se señala en otro artículo de esta publicación. ¿Qué es lo que asegura que una política pública sea pertinente, trascienda a la mera formulación y se implemente? ¿Qué es lo que asegura que una vez implementada se logren los resultados esperados y se protejan así los derechos de los ciudadanos para lo cual se elaboró esa política?

El postulado central de este ensayo es que desde el diseño de una ley, una política pública y el logro de su propósito, intervienen necesariamente los fundamentos sobre los cuales se construyó esa política, su pertinencia a las necesidades que le dieron origen y las estructuras que la crean y/o las que deben hacerse responsables no sólo de acatar la ley y de convertirla en una política operativa sino de asegurar que esa operatividad se concrete en logros demostrables en los plazos esperados y con los recursos necesarios. Mientras más alcance deba tener esa política pública en términos de su espectro de acciones, de tiempo, de población y de ámbito geográfico, mayor es la necesidad de coincidencia entre estructuras de diversa índole que, para ello, deben ser convergentes y sinérgicas. Así, a mayor trascendencia de la política, más necesaria es la convergencia de estructuras, no sólo de gobierno sino de Estado.

Aun cuando podría parecer obvio, es necesario decir que a lo largo de este documento estará siempre como telón de fondo la protección de los derechos ciudadanos, a quienes se dirigen los esfuerzos legislativos y de gobierno.

Es por ello importante analizar el tema de la protección de los derechos ciudadanos a partir de una descripción de la naturaleza de las políticas públicas para seguir con la dinámica del sistema de salud que se ve inevitablemente influido por esas políticas, y concluir con la identificación de estructuras gubernamentales y de Estado responsables de la creación de esas políticas y de su ejecución. En este caso, el énfasis se hace en el Consejo de Salubridad General, una estructura que, por sus características peculiares como órgano del Estado y no de gobierno, debe permitir una amplia convergencia para establecer medidas efectivas para la defensa del derecho a la protección de la salud.

\section{La naturaleza de las políticas públicas}

Como se mencionó anteriormente, en México las políticas tendentes a evitar los daños producidos a la salud a causa del tabaquismo no tuvieron mayores efectos en el pasado, a pesar incluso de que surgieron de diversas fuentes, tal como lo ha demostrado Éctor Jaime Ramírez-Barba a través de la amplia revisión que se presenta en este mismo número. Por ello, tal vez sea interesante iniciar el análisis de la naturaleza de las políticas públicas identificando algunas de las características que impiden que una política pública logre su cometido. En el rastreo de lo acontecido a las políticas hasta ahora para proteger a los no fumadores puedan encontrarse causas que, si se evitan, pueden definir las características deseables de una política pública. Se citarán solamente diez causas posibles:

1. La política pública no responde a una prioridad precisa y evidente. En efecto, cuando no existe el fundamento en los hechos que demuestre la necesidad de esa política y los aspectos que ésta debe considerar, es muy probable que la política fracase. El blanco no está claro y, por consiguiente, es muy probable que el disparo yerre.

2. La voluntad política para impulsar las medidas que se pretende establecer mediante una política pública es mínima o inexistente. Si esto ocurre, no hay manera de dar respaldo a decisión alguna.

3. Baja percepción social de la prioridad de la política. Es posible que la política pública se estime prioritaria por quienes desean impulsarla, e incluso que la voluntad política sea alta en un principio, sin embargo, si la percepción de la sociedad no coincide con esa estimación, será difícil su aceptación y, por consiguiente, su implantación. Más aún, si esta percepción negativa se manifiesta con intensidad, seguramente la voluntad política será neutralizada y muy probablemente modificada o incluso extinguida.

4. Pobre argumentación sobre la necesidad y los beneficios de la política en cuestión. Aun cuando pudiesen existir los fundamentos suficientes, es necesario plantear correctamente los argumentos que hagan evidente la necesidad de una determinada política. Es muy probable que ante una baja percepción social de la necesidad de una política, si la argumentación se hace de manera inteligente y contundente, esa percepción pueda ser modificada.

5. Formulación imprecisa. Como cualquier estrategia, una política pública debe ser lo suficientemente clara en cuanto a los objetivos concretos que se persiguen, de ser posible, susceptibles de ser medidos; los tiempos 
en los que se espera sean logrados; y las medidas que deben tomarse para alcanzarlos. De no ser así, a pesar de cualquier argumentación intachable, de una alta percepción social favorable, voluntad política, y de una elevada pertinencia a una situación determinada, será imposible implantarla.

6. Respaldo jurídico inadecuado. Todas las situaciones mencionadas anteriormente pueden ser superadas, sin embargo, si jurídicamente se carece del sustento adecuado la política naufragará rápidamente en las aguas de su propia fragilidad.

7. No existencia de incentivos y/o penalizaciones acordes a la trascendencia que se le quiere dar a la política pública. Tan importantes son unos como las otras. La voluntad humana se orienta en función de ambas.

8. Insuficiencia de recursos para implantarla. Aun cuando esto parece obvio, con frecuencia se pierde de vista que la instrumentación de cualquier política pública requiere de los recursos necesarios para hacerla efectiva. Ello, debe decirse, no asegura que la política tenga éxito como a veces se piensa, aunque sin éstos es imposible.

9. Falta de seguimiento a la ejecución apoyado en estructuras específicamente responsables de éste. Es también muy frecuente observar que una vez emitida una política pública se asuma que por el mero hecho de hacerla del conocimiento de todos se cumplirá inevitablemente. Sin un plan de acción que permita un seguimiento puntual de los avances trazados y una clara definición de responsabilidades para que esto ocurra es imposible que la política pública se haga realidad o se concrete como se espera.

10. Falta de evaluación de impactos. Más allá del seguimiento puntual, en tanto no se evalúen las políticas públicas, su ejecución puede ser una mera ilusión.

Como se aprecia, cada una de las causas mencionadas puede convertirse en una característica de éxito si se plantea positivamente. Lavis, ${ }^{1}$ además, considera que la efectividad de las políticas públicas depende de las características de las decisiones que constituyen esas políticas, de quién toma esas decisiones, del contexto en el que se dan y de la evidencia que se construye para fundamentarla.

Esta última característica merece ser destacada en el tema que atañe al ensayo pues, en el que caso de la protección de los no fumadores, la evidencia se ha ido acumulando a lo largo de los años y, a pesar de ello, tal vez no había sido utilizada como hasta ahora. En efecto, ante las enormes resistencias que el tema ha generado, solamente una demostración contundente de los daños causados y de los beneficios esperados podía contrarres- tarlas. Sin embargo, el fundamento construido sobre la evidencia científica no ha sido común en estos casos. Si bien en el ejercicio de la medicina se ha incrementado la consciencia sobre la importancia de esta evidencia para la toma de decisiones clínicas desde los años ochenta, Lemieux ${ }^{2}$ deja en claro que ello no ha ocurrido en la misma medida para la toma de decisiones de alta dirección y mucho menos para la definición de políticas públicas. Esta política para la defensa de los no fumadores es un buen ejemplo de sustentación sobre bases firmes.

Si bien en la praxis por lo menos esas 10 causas y las características que propone Lavis son importantes, lo es también tener un concepto claro de la naturaleza y de la génesis de las políticas públicas. Son muchas las definiciones que pueden ser encontradas y elaboradas. Una de las más pertinentes para este trabajo define a la política pública como "un instrumento [...] mediante el cual se pretende alcanzar desde el Estado, en forma sistemática y coherente, ciertos objetivos de interés para el bienestar de toda la sociedad civil". ${ }^{3}$ Podestá, el autor de esta definición dice, además, que "desde un punto de vista metodológico la política pública debe originarse y formularse en forma sistemática y coherente, tratando de coordinar el tema espacial (internacional, nacional, regional, comunal, micro-local), con el tema temporal (corto, mediano y largo plazo), considerando además su viabilidad política y financiera".

Por otra parte, la gestación de una política pública puede ser explicada desde varias perspectivas. Una, explica la generación de una política pública como un proceso lineal e incremental en el que ésta se va gestando paulatinamente desde un "agente originador" que la impulsa y avanza en la medida de lo posible. Otra, como un proceso de interacción pública entre legisladores, funcionarios públicos, grupos de interés, población en general, a través del cual se logra un acuerdo de intereses y balances de poder. Otra más, como un proceso dialéctico resultante de conflictos permanentes que se confrontan para llegar a una conclusión, posiblemente temporal. ${ }^{4}$ Ninguna de las tres excluye a las otras dos, lo cual hace más complejo aún dilucidar la génesis de una política pública particular. Cualquiera que sea la perspectiva desde la cual se analice la génesis de una política pública que se produzca en un entorno con mínimas características democráticas manifiesta que se trata de un proceso y no de decisiones aisladas, de un proceso frecuentemente complicado por la cantidad de interacciones involucradas, y que podría estar siempre inconcluso por requerir ajustes de manera prácticamente permanente.

En resumen hasta aquí, en la defensa del derecho a la protección de la salud, en este caso mediante una política que protege a los no fumadores, será necesario tener en cuenta cómo se ha gestado ésta, las caracterís- 
ticas que hasta ahora le han permitido salir adelante y las que deban ser consideradas cuidadosamente para asegurar su éxito. No es materia de este ensayo, sin embargo, hacer un análisis puntual de esta política, dado que la perspectiva histórica que permita un juicio mucho más objetivo es prácticamente inexistente.

\section{La dinámica de los sistemas de salud}

El conjunto de recursos que producen servicios de salud, que deben ser accesibles, equitativos, eficientes y de calidad, y por supuesto utilizados por la población, es a lo que puede considerarse como sistema de salud. Las múltiples variables que intervienen en su conformación y funcionamiento en un momento determinado hacen de éste una organización compleja que, por otra parte, evoluciona constantemente en función de la dinámica demográfica, epidemiológica, tecnológica y cultural que constituye su entorno más relevante. Ni qué decir de las variables económicas y políticas que lo influyen. Pero, además, es imposible imaginar un sistema de salud sin las políticas públicas que definan no sólo las características de la oferta de los servicios y su financiamiento, sino también las necesidades que deben ser atendidas.

El entorno actual de los sistemas de salud es particularmente dinámico. En efecto, baste citar lo que podemos denominar la "rima de las revoluciones" que en años recientes y en los próximos imprimirán una imagen seguramente muy diferente a cualquier sistema de salud: genómica, proteómica, informática, telemática, robótica, tecnología nanoscópica, bioética, por citar sólo algunos de los cambios más rápidos que se viven. A ello debe agregarse el profundo cambio demográfico y epidemiológico que podría ser también considerado parte de estas revoluciones en tanto que nunca la población de este país había envejecido como ahora lo hace y lo hará, y jamás se había encontrado ante una situación epidemiológica caracterizada por la amenaza de una epidemia de padecimientos crónico-degenerativos.

Otras variables que han modificado y seguirán modificando la naturaleza de los sistemas de salud, y el mexicano no escapa, es la creciente exigencia de la población a tener servicios con altos niveles de calidad y seguridad; la mayor presencia de los medios de comunicación en los asuntos de la atención a la salud; el gran acceso a información sobre atención a la salud a través de Internet, para expertos y legos; o el incremento de las medicinas complementarias, por citar otras más.

Esta situación exige que las políticas públicas sean oportunas, bien enfocadas a los problemas que deben resolver, y pertinentes no sólo para enfrentar sino también para prever las posibles consecuencias de este entorno altamente dinámico. Ante un blanco móvil, la mira debe anticipar el movimiento y el disparo debe ser hecho con firmeza y con certeza. Solamente así será posible cuidar adecuadamente el derecho a la protección de la salud de la ciudadanía. Lo que hemos atestiguado a lo largo de la historia del último siglo en el caso de la protección a los no fumadores es que, por la razón que haya sido, la mira se apuntó pero el disparo finalmente no tuvo efecto y el fenómeno y los daños que debían prevenirse siguieron creciendo.

\section{Las estructuras del Estado}

Incluso cuando la formulación de las políticas públicas es el punto de partida indispensable y las necesidades de la población y la protección de sus derechos el objetivo, ambos aspectos son dos extremos que se unen solamente mediante las estructuras organizacionales que permiten articular unas con los otros de manera que incidan en el comportamiento del sistema de salud para que responda como se considere necesario que lo haga.

Enfrentar los múltiples retos que se plantean a los sistemas de salud requiere no sólo de la respuesta de las instituciones de atención a la salud sino de un enfoque mucho más amplio. En efecto, problemas como la obesidad, la diabetes, la depresión o el tabaquismo (entre otras adicciones), como es el caso que atañe a esta publicación, rebasan las posibilidades del sistema de salud y requieren de la participación de otras instancias gubernamentales y no gubernamentales. Para prevenirlos y resolverlos son necesarias verdaderas políticas de Estado en las que intervengan todos los actores que deban participar. Ello implica que se cumplan las características ya mencionadas que hagan posible que una política pública tenga el efecto deseado, pero también que existan las instancias de convergencia de los actores al más alto nivel posible. Tal es el caso, entre otras estructuras, del Consejo de Salubridad General.

Creado en 1841, el Consejo de Salubridad General adquirió rango constitucional en 1917, mediante su incorporación en el Artículo 73, Fracción XVI de la Carta Magna, que a la letra dice: "dependerá directamente del Presidente de la República, sin intervención de ninguna Secretaría de Estado, y sus disposiciones generales serán obligatorias en el país". En lo que corresponde a la protección de la salud de los no fumadores, una de las varias atribuciones del Consejo se precisa en el artículo mencionado: "Las medidas que el Consejo haya puesto en vigor en la campaña contra el alcoholismo y la venta de sustancias que envenenan al individuo o degeneran la especie humana, así como las adoptadas para prevenir y combatir la contaminación ambiental, serán después revisadas por el Congreso de la Unión en los casos que le competan". 5 
Dada la constitución del Consejo de Salubridad General, en el que participan once secretarías de Estado, las principales instituciones públicas de atención a la salud, secretarios de Salud de los estados, universidades, academias, colegios y asociaciones privadas, el Consejo constituye un verdadero punto de convergencia estratégica para la promoción de políticas de Estado. La Ley General de Salud define al Consejo como autoridad sanitaria como lo es también el Presidente de la República y la Secretaría de Salud. ${ }^{6}$ De esta manera, queda explícita la autoridad del Estado, en este caso representado por el Consejo, y la autoridad del Gobierno, representado por la Secretaría de Salud, y ambas atribuciones encabezadas por el propio Presidente de la República en su carácter de jefe de Estado y de Gobierno.

El Consejo de Salubridad General publicó en 2004 el "Acuerdo por el que se emite recomendación a fin de proteger la salud de los no fumadores por la exposición involuntaria al humo del tabaco". 7 El efecto de dicho Acuerdo no fue, sin embargo, el esperado. Ello se debió probablemente, entre otras razones, al hecho de que no existe en el Consejo una estructura responsable de dar seguimiento a sus acuerdos, con lo cual está ausente uno de los requisitos indispensables para que las políticas públicas se concreten.

Hoy, la convergencia para la definición de las políticas de Estado exige no sólo de las instancias gubernamentales sino también de la participación de la sociedad civil y, por supuesto, del Poder Legislativo. La iniciativa de ley para la protección de los no fumadores partió ahora y por primera vez del Poder Legislativo. La instrumentación, sin duda, es responsabilidad del Poder Ejecutivo. Será la Secretaría de Salud responsable de que se transite desde la formulación de una ley hasta la ejecución de una política pública. Deberemos seguir perfeccionando este ejercicio de articulación entre generación, formulación, reglamentación y ejecución de las políticas públicas, en las cuales todas las estructuras que configuran el Estado mexicano, incluido el Consejo de Salubridad General, converjan para proteger los derechos de los ciudadanos, en este caso, el derecho a la protección de la salud.

\section{Conclusión}

Cualquier esfuerzo que se haga para proteger los derechos ciudadanos, debe propiciar la convergencia de todos los actores que deban estar involucrados. Por su naturaleza, las políticas públicas que se dirigen hacia la protección de la salud adquieren hoy una enorme trascendencia ante la dinámica del sistema de salud y de la sociedad. Por ello, es cada vez más importante construir políticas de Estado que reflejen esa convergencia.

El Consejo de Salubridad General se fortalece y rescata su vocación primigenia para responder a esa expectativa, como una de las estructuras del Estado que debe contribuir, con otras más, a la articulación de la génesis, formulación y ejecución de políticas públicas que cumplan con las características necesarias para que las necesidades de la población y la voluntad política en defensa de los derechos ciudadanos transiten de un desideratum hacia una realidad de efectos positivos demostrables.

\section{Referencias}

I. Lavis JN. A political science perspective on evidence-based decision making. En: Lemieux-Charles L, Champagne F. Using knowledge and evidence in health care. Toronto: University of Toronto Press, 2004:7085.

2. Champagne F, Lemieux-Charles L, McGuireW. Towards a broader understanding of the use of knowledge and evidence in health care. En: Lemieux-Charles L, Champagne F. Using knowledge and evidence in health care. Toronto: University of Toronto Press. 2004:3-I7.

3. Podestá J. Problematización de las políticas públicas desde la óptica regional. Última Década 200I;15:163-175.

4. Doern B, Aucoin P. Public policy in Canada. Toronto, Ont.: Gage Publishing Limited, 1979.

5. Constitución Política de los Estados Unidos Mexicanos. Artículo 73, Fracción XVI. México, 2007.

6. Ley General de Salud. Artículo 4. México, 2007

7. Consejo de Salubridad General. Acuerdo por el que se emite recomendación a fin de proteger la salud de los no fumadores por la exposición involuntaria al humo de tabaco. Diario Oficial de la Federación 28 de mayo. México: Secretaría de Gobernación, 2004. 